Review Article

\title{
A New Era of Submillimeter GRB Afterglow Follow-Ups with the Greenland Telescope
}

\author{
Yuji Urata, ${ }^{1,2}$ Kuiyun Huang, ${ }^{3}$ Keiichi Asada, ${ }^{2}$ Hiroyuki Hirashita, \\ Makoto Inoue, ${ }^{2}$ and Paul T. P. Ho ${ }^{2,4}$ \\ ${ }^{1}$ Institute of Astronomy, National Central University, Chungli 32054, Taiwan \\ ${ }^{2}$ Academia Sinica Institute of Astronomy and Astrophysics, Taipei 106, Taiwan \\ ${ }^{3}$ Department of Mathematics and Science, National Taiwan Normal University, Linkou District, New Taipei City 24449, Taiwan \\ ${ }^{4}$ Harvard-Smithsonian Center for Astrophysics, 60 Garden Street, Cambridge, MA 02138, USA
}

Correspondence should be addressed to Yuji Urata; urata@astro.ncu.edu.tw

Received 1 December 2014; Revised 19 March 2015; Accepted 23 March 2015

Academic Editor: Valery Nakariakov

Copyright (C) 2015 Yuji Urata et al. This is an open access article distributed under the Creative Commons Attribution License, which permits unrestricted use, distribution, and reproduction in any medium, provided the original work is properly cited.

\begin{abstract}
Planned rapid submillimeter (submm) gamma-ray-bursts (GRBs) follow-up observations conducted using the Greenland Telescope (GLT) are presented. The GLT is a $12-\mathrm{m}$ submm telescope to be located at the top of the Greenland ice sheet, where the high altitude and dry weather porvide excellent conditions for observations at submm wavelengths. With its combination of wavelength window and rapid responding system, the GLT will explore new insights on GRBs. Summarizing the current achievements of submm GRB follow-ups, we identify the following three scientific goals regarding GRBs: (1) systematic detection of bright submm emissions originating from reverse shock (RS) in the early afterglow phase, (2) characterization of forward shock and RS emissions by capturing their peak flux and frequencies and performing continuous monitoring, and (3) detections of GRBs at a high redshift as a result of the explosion of first generation stars through systematic rapid follow-ups. The light curves and spectra calculated by available theoretical models clearly show that the GLT could play a crucial role in these studies.
\end{abstract}

\section{Introduction}

Gamma-ray bursts (GRBs) are among the most powerful explosions in the universe and are observationally characterized according to intense short flashes mainly in highenergy band (so-called "prompt emission") and long-lasting afterglows observed in X-ray to radio bands. Both types of radiation are sometimes extremely bright and can be observed using small- and middle-aperture optical and nearinfrared telescopes (e.g., $[1,2]$ ). Because of their intense luminosity, the highest redshift $(z)$ in the reionization epoch $(z \gtrsim 8)$ has been observed and has a high possibility for discovery even at $z>10$ [2]. Although there are various diversities (long/short duration of prompt $\gamma$-ray radiation, $\mathrm{X}$-ray flares associated with X-ray afterglows, and complex temporal evolutions of afterglows), optical afterglow and host galaxy observations indicate that the majority of longduration GRBs occur as a result of the death of massive stars (e.g., [3]). Thus GRBs are unique and powerful means of observing explosions first generation stars (population III, POP-III). Understanding the diversity of the astrophysical entities that cause GRBs is the subject of ongoing study and represents one of the most prominent inquiries in modern astrophysics.

Using GRBs to investigate the high- $z$ universe requires an understanding of their radiation mechanisms. Confirming the existence of reverse shocks (RS) and ascertaining their typical occurrence conditions are critical. The GRB afterglow is believed to involve a relativistically expanding fireball (e.g., [4]). The Interstellar Medium (ISM) influences the fireball shell after it has accumulated, and considerable energy is transferred from the shell to the ISM. The energy transfer is caused by two shocks: a forward shock (FS) propagating into the ISM and an RS propagating into the shell. Millimeter/submillimeter $(\mathrm{mm} / \mathrm{submm})$ observations are the key elements in understanding the emission mechanism of GRB 
afterglows. They provide "clean" measurements of source intensity and are unaffected by scintillation and extinction. Hence, studies of submm properties of the afterglow are likely to enrich the understanding of GRB physics.

In this paper, we review the status and achievements of submm afterglow observations in Section 2. In Section 3, we introduce the Greenland Telescope (GLT) project and its advantages for GRB follow-up observations. We also expect the GRB follow-ups in the era of GLT in Section 4. On the basis of these advantages, we establish three scientific goals by introducing the expected light curves and spectra with the expected sensitivity limit of GLT in Section 5. Finally, we summarize this paper in Section 6.

\section{Status and Achievements of Submillimeter Afterglow Follow-Ups}

Numbers of dedicated follow-up instruments of GRBs and afterglows have been developed in $\gamma$-ray, X-ray [5], optical, and near-infrared (e.g. [6-12]). Afterglow observations in X-ray and optical have been adequately covered from very early phase including some fractions of $\gamma$-ray prompt phase (e.g., [13-16]). In addition, more than 300 afterglow observations have been made at the $\mathrm{cm}$ wavelengths mainly using the Very Large Array (e.g., [17]). However, submm has lagged behind X-ray and optical. Figure 1 shows a schematic summary of achievements in GRB observations according to wavelength and time range. It is obvious that prompt afterglow observations are lacking at submm wavelengths. The numbers of submm-detected events have been limited as summarized in Figures 1 and 2. Figure 2 shows all of afterglow observations in submm bands (230 and $345 \mathrm{GHz}$ ) including upper limits. There have been only seven detections and three well-monitored events (GRB030329, GRB100418A, and GRB120326A) in the submm bands. Unlike X-ray and optical observations, afterglow monitoring in the submm band covers only the late phase of GRBs and misses their brightening phases. However, several of these observations, in conjunction with intensive X-ray and optical monitoring, as in the GRB 120326A case [18], have addressed crucial physical properties of afterglow. Hence, submm afterglow observations are crucial for understanding the nature of GRBs. In the following, we briefly summarize three wellmonitored submm afterglow cases.

GRB030329. Because of the low redshift $(z=0.168)$ origin and bright optical afterglow ( 13 mag at 0.1 days), numerous follow-up observations were conducted at various wavelengths (e.g., [3, 19-31]). The 250-GHz monitoring follow-ups were performed by the MAMBO- 2 bolometer array on the IRAM 30-m telescope [32] and the IRAM Plateau de Bure Interferometer (PdBI) [33]. The monitoring observations were conducted from 1.4 to 22.3 days after the burst. The light curve after $\sim 8$ days exhibited a simple power-law decay with a decay index of -1.68 [32]. The value was consistent with that determined for the optical decay after $\sim 0.5$ days and indicated a common physical effect [34]. These monitoring observations supported the two-component jet model, in which a narrow-angle jet is responsible for the high-energy emission and early optical afterglow; the radio afterglow emission is powered by the wide-angle jet $[32,35]$.

GRB100418A. The Submillimeter Array (SMA, [36]) was used to observe the submm afterglow from $\sim 16$ hours after the burst. Continuous monitoring proceeded until 5 days after the burst, at which point it became undetectable [37]. As shown in Figure 2, the submm light curve exhibited a significant fading (equivalent decay power-law index of $\sim-1.3$ ) between 1 and 2 days and then exhibited a plateau phase until 4 days. The X-ray and optical light curves showed a late bump peak at $\sim 5 \times 10^{4} \mathrm{~s}[38]$.

GRB120326A. The SMA observation provided the fastest detection to date among the seven submm afterglows at $230 \mathrm{GHz}$ (Figure 2). In addition, comprehensive monitoring in the X-ray and optical bands was also performed. These observations revealed that the temporal evolution and spectral properties in the optical bands were consistent with the standard FS synchrotron with jet collimation $\left(6^{\circ} .7\right)$. Furthermore, the X-ray and submm behavior indicated different radiation processes from the optical afterglow as shown in Figure 3. Introducing synchrotron self-inverse Compton radiation from $\mathrm{RS}$ is a possible solution that is supported by the detection and slow decay of the afterglow in the submm band. As shown in Figure 4, the light curve exhibited the slow temporal evolution $\left(\alpha_{\text {submm }}=-0.33\right)$ between $4 \times 10^{4}$ and $1 \times 10^{5} \mathrm{~s}$; this evolution is consistent with the expected decay index of the RS with the $v_{\mathrm{obs}}<v_{m, \mathrm{RS}}^{\text {Sync }}$ condition [18]. Here, $v_{\text {obs }}$ and $\nu_{m, \text { RS }}^{\text {Sync }}$ are the observing frequency and the characteristic synchrotron frequency of the RS. And because half of the events exhibit similar X-ray and optical properties (e.g., [39-41]) as the current event, GRB120326A constitutes a benchmark requiring additional rapid follow-ups conducted using submm instruments such as the SMA and the Atacama Large Millimeter/submm Array (ALMA).

To enhance afterglow studies, submm monitoring observations from an early phase are required. Although rapid follow-ups have been performed using the SMA, as in the GRB130427A case (beginning 1.6 hours after the burst), these follow-ups have been still limited; specifically, we have failed to detect the GRB130427A afterglow with an insufficient sensitivity $(\sim 10 \mathrm{mJy})$ for detecting the RS component [42$44]$ caused by poor weather conditions. The ongoing SMA programs have also been suffering from the poor weather conditions of the Mauna Kea site. The condition of the Mauna Kea site for the SMA and the James Clerk Maxwell Telescope (JCMT) is inferior for submm observations comparing with other sites such as the ALMA site. Five out of 13 observations with SMA (average responding time is 11.3 hours) were made under the poor or marginal weather conditions. Hence, weather condition for submm bands is one of the crucial points for the time critical observations. As demonstrated through JCMT observations [45-50], rapid follow-ups can be managed by using existing submm telescopes with suitable follow-up programs and observation system. The typical delay time of JCMT is hours scale (average of $59 \mathrm{~min}$ with 


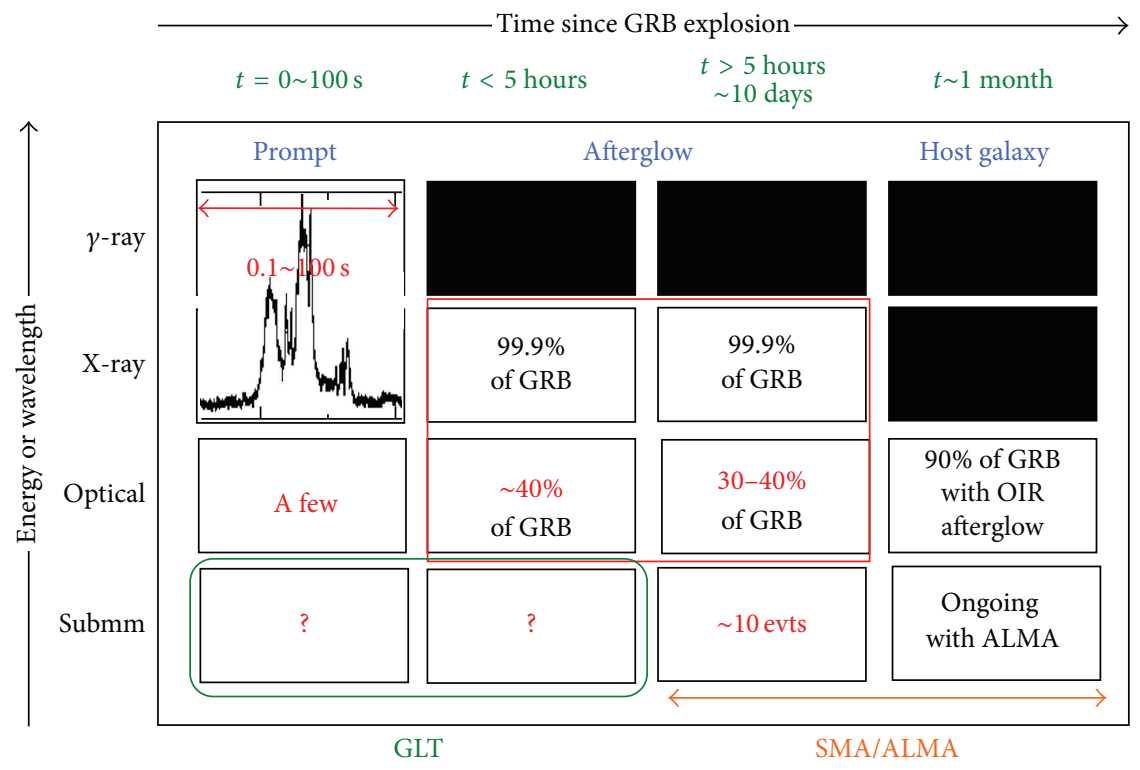

(a)

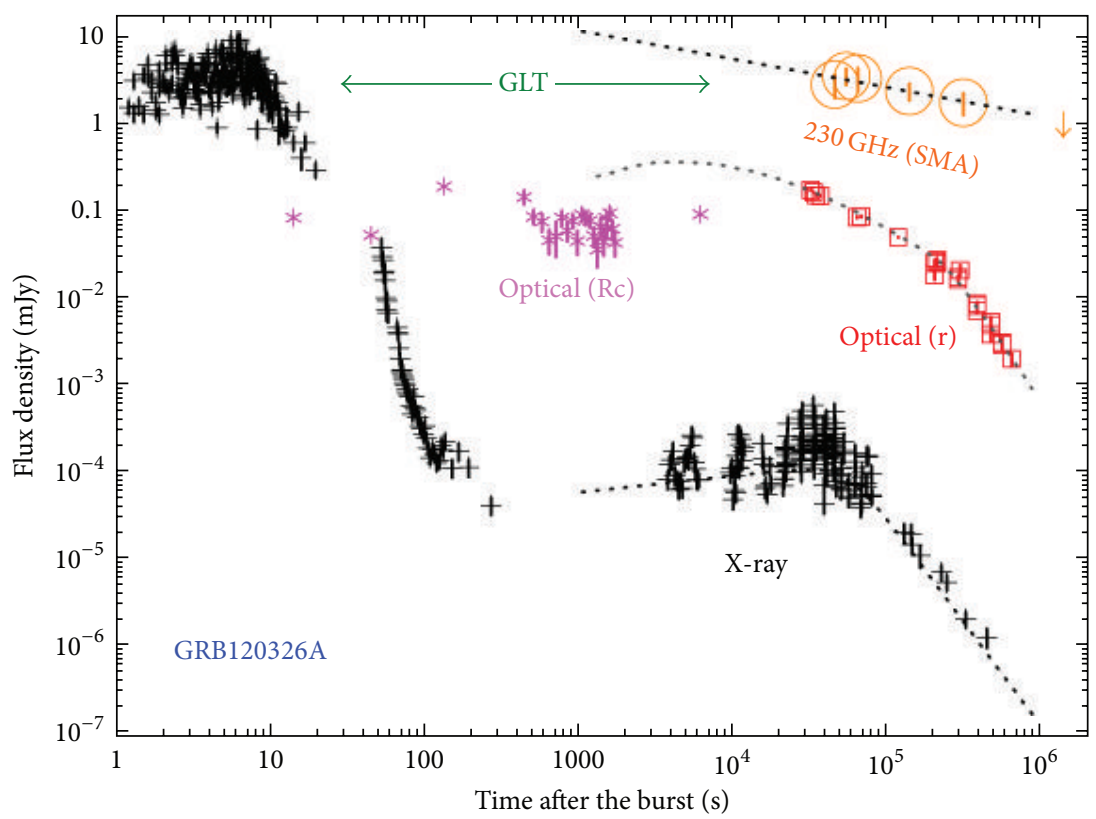

(b)

FIGURE 1: (a) Schematic summary of the GRB observational achievements along with time from the bursts in individual wavelength (from $\gamma$-ray to submm). (b) One of actual light curves in X-ray, optical, and submm with the earliest submm detection.

6 GRB observations). Therefore, the succession of the JCMT rapid response system is also desired in the future. In addition, the constructions of dedicated submm telescopes based on these experiences at the better observing site are required to conduct systematic rapid and dense continuous follow-ups (sometimes coordinated with several submm telescopes at the different longitude for covering light curve within a day).

\section{Greenland Telescope}

The GLT is a state-of-the-art $12-\mathrm{m}$ submm telescope to be located in the Summit Station in Greenland. The aims of the project are establishing a new submm very long baseline interferometer (VLBI) station for the first imaging of shadow of supermassive black holes in M87 [51] and exploring a new terahertz frequency window [52] and time-domain astronomy in submm (e.g., this paper). The expected first light of the GLT will be made in 2017/18.

3.1. Site of the Greenland Telescope. The Summit Station is located on top of the Greenland ice sheet, at $72.5^{\circ} \mathrm{N}$ and $38.5^{\circ} \mathrm{W}$ (north of the Arctic Circle) at a 3,200-m altitude. The temperature is extremely low, especially in winter when 


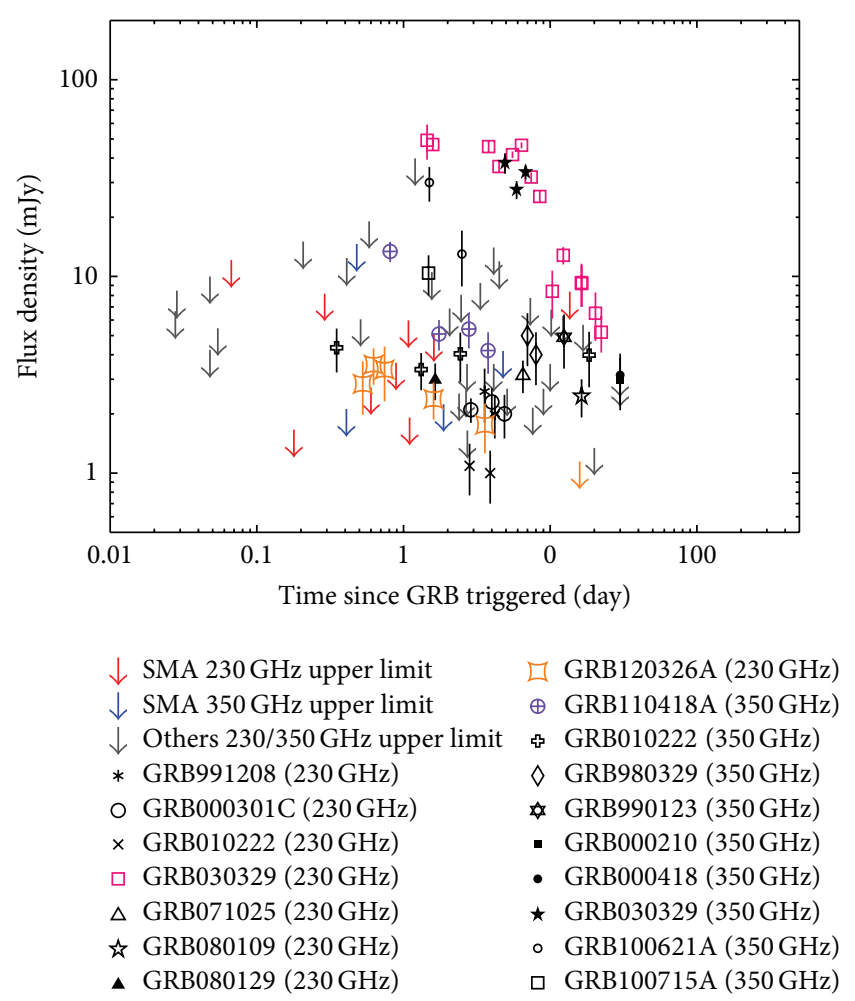

FIGURE 2: Light curve summary of afterglow observations in submm bands (230 and $345 \mathrm{GHz}$ ). Red (230 GHz upper limits), blue ( $345 \mathrm{GHz}$ upper limits), orange (GRB120326A), and purple (GRB100418A) points were obtained with the SMA. The SMA successfully monitored two of three well-observed submm afterglows, GRB100418A and GRB120326A.

the temperature reaches between $-40^{\circ} \mathrm{C}$ and $-60^{\circ} \mathrm{C}$ (the lowest temperature of $-72^{\circ} \mathrm{C}$ has been recorded). Because of the combination of low temperature and high altitude, considerably low opacity is expected. In 2011, a tipping radiometer at $225 \mathrm{GHz}$ was deployed to Greenland to monitor the sky and weather conditions at the Summit Station and measure opacities from October 2011 to March 2014. The best and the most frequent opacities at $225 \mathrm{GHz}$ were 0.021 and 0.04 , respectively [51]. The weather conditions are compatible with those at the ALMA site and significantly better than those of the Manna Kea site [53]. These low opacities and weather conditions are the advantage of the GLT in managing submm and time critical observations including GRB followups with higher sensitivity (or short exposure cycle).

3.2. Planned Instruments and Expected Sensitivities. The GLT will be initially equipped with VLBI receivers at 86, 230, and $345 \mathrm{GHz}$. Whether large single-dish receivers as secondgeneration instruments (e.g., submm heterodyne arrays, a $\mathrm{THz} \mathrm{HEB}$ array, and bolometric spectrometer array) will be installed depends on the scientific merits of the instruments and is still under discussion. For GRB observations, three frequency bands of the VLBI receivers are appropriate and, therefore, the first generation receivers can be used for afterglow observations. The current expected sensitivities of VLBI receivers are 36 and $88 \mathrm{mJy}$ with $1 \mathrm{~s}$ integration at 230 and $345 \mathrm{GHz}$, respectively [54]. These are at least two times better than those of SMA and JCMT. We note that minimum integration time would be less than $0.5 \mathrm{~s}$, since duty cycle for positional switch will be $2 \mathrm{~Hz}$. Longer integration will be achieved as the accumulation of the short integrated data points. Hence, the GLT with the receivers will provide sufficient sensitivities to detect GRB counterparts at the $230 \mathrm{GHz}$ band with shorter exposure (e.g., $3 \sigma$ limit of $48.3 \mathrm{mJy}$ with $5 \mathrm{~s}, 10.8 \mathrm{mJy}$ with $100 \mathrm{~s}, 3.6 \mathrm{mJy}$ with $15 \mathrm{~min}$, and $1.8 \mathrm{mJy}$ with $1 \mathrm{~h}$ ). This shorter exposure cycle is one of advantages to characterized temporal evolutions of submm afterglows. The field-of-view (FOV) of the receivers will be $25^{\prime \prime}$ and $16^{\prime \prime}$ at 230 and $345 \mathrm{GHz}$, respectively. These relatively narrow FOV require a tiling observation for covering entire error region determined by hard X-ray instruments (e.g., Swift/BAT) or accurate position determination with X-ray afterglows.

We are also planning to install semiautomated responding system for the GRB alerts to manage the rapid GRB follow-ups with secure procedures at the extreme site. We enjoyed the prototype system at the Kiso observatory [23]. The pointing will be started using the position determined by $\gamma$-ray instruments and additional adjustment will be made responding to the position of X-ray afterglows. By combination use of this system and site advantages including visibility for targets as shown in Figure 5, we will be able to perform nearly real-time follow-ups for GRBs whose declinations are higher than 30 degrees. A continuous monitoring (nearly video mode) will be managed in the first one day.

\section{Expected GRB Follow-Up Observations in the GLT Era}

To achieve successful observations, rapid follow-ups using the GLT will be coordinated through Swift and the planned Space-based Multiband Astronomical Variable Objects Monitor (SVOM) [55]. One of the obstacles to performing rapid follow-ups of Swift-detected GRBs is the poor visibility from ground-based instruments. Although Swift has enabled detecting between 100 and 150 events per year, only 10 GRBs per year are observable from the major astronomical observation sites (e.g., Mauna Kea, Chile) without any delay from their $\gamma$-ray triggers, because of visibility problems that occur when using ground-based instruments and the random pointing strategy associated with Swift observations. According to statistical data in 10 years of Swift observations, 10 to 12 events per year could be observed from the early phase of the afterglow by using currently existing telescopes within a $0-3$-hour delay by maintaining a proper exposure time (>3-4 hours). The current fraction of total Swift/BAT pointing time to around antisun directions (sun hour angle from 9 to 15 hours) is limited to $30-40 \%$ [56]. The ideal location of the GLT will enable solving this problem. As shown in Figure 5, GRBs located at a declination higher than 29 degrees will always exhibit an elevation angle higher than 12 degrees over days. Hence, in winter, the GLT will be able to begin rapid GRB follow-ups without any delay 


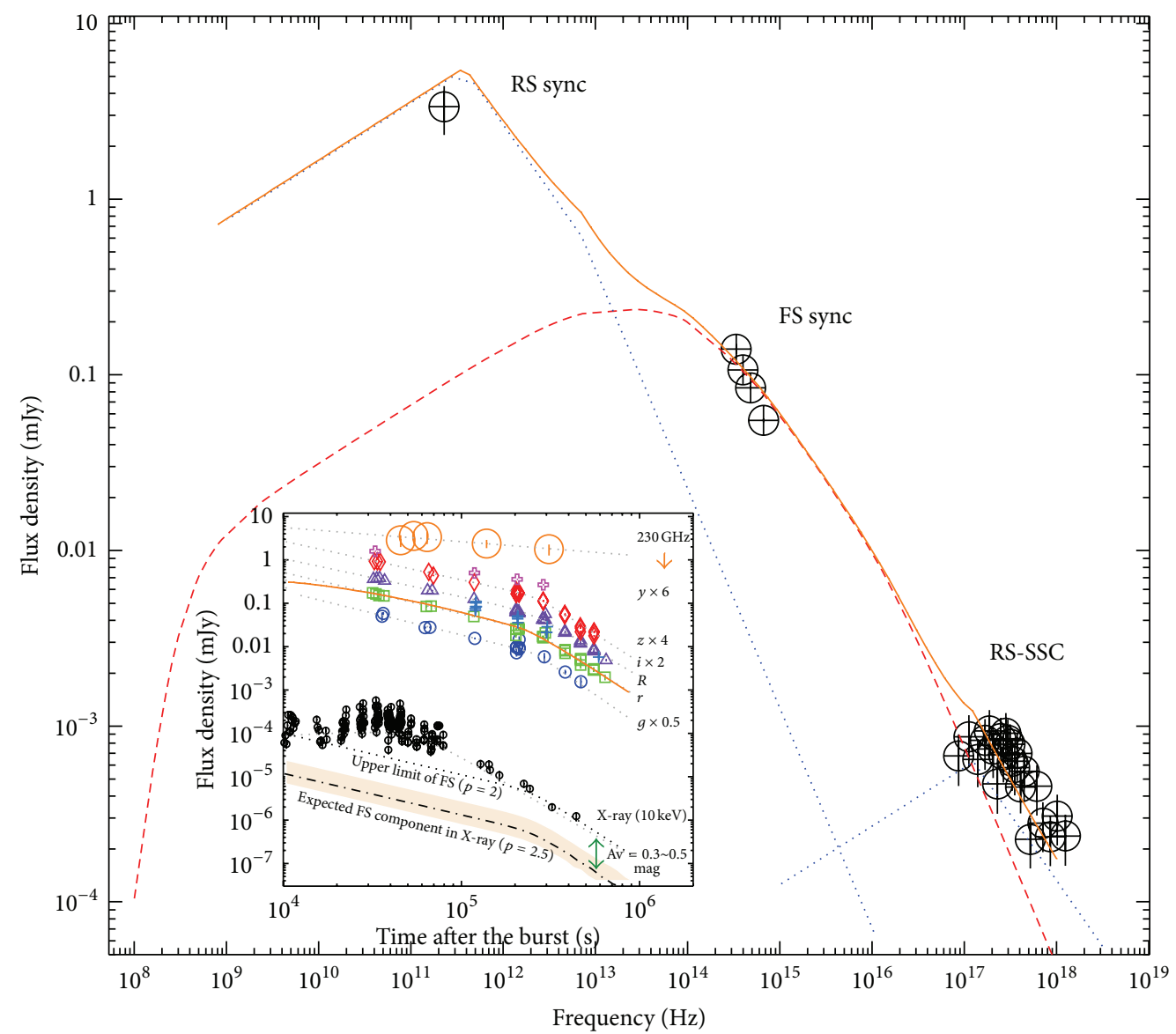

FIGURE 3: Main panel: The spectral energy distribution of GRB120326A at $6.42 \times 10^{4} \mathrm{~s}$ after the burst [18]. The red dashed line shows the forward shock synchrotron model spectrum calculated using the boxfit code [89] with the same parameters for the best modeling light curve shown in the subpanel. The blue dotted lines show the reverse shock synchrotron radiation and its self-inverse Compton component calculated based on Kobayashi et al. [72] using the observed values and model function for the forward shock component. Subpanel: X-ray, optical, and submm light curves of the GRB120326A afterglow. The grey dotted lines show the best analytical fitted functions described in the text. The orange solid line shows the best modeling function for the $r$-band light curve obtained with the numerical simulation using boxfit.

caused by unfavorable visibility and perform continuous submm monitoring over days. In addition, these observation conditions are advantageous for observing GRB afterglows that exhibit a rich diversity in various time ranges. In summer, the $86 \mathrm{GHz}$ receiver will be used when weather conditions are unsuitable for observations at 230 and $345 \mathrm{GHz}$.

SVOM (2020 ) will be a timely mission for conducting rapid GLT observations. The GRB detectors will observe antisun directions that enable ground-based instruments to begin follow-ups and continuous long-term monitoring of markedly early cases immediately after receiving GRB alerts. The GRB detection rate of SVOM will be $\sim 80$ events per year, providing 10 to 20 events per year for rapid GLT followups. In a typical GRB case, X-ray afterglows will be observed immediately through a SVOM X-ray telescope (MXT) [57], with the same strategy of X-ray observations of Swift. This provides a position accuracy of the counterpart of $2-3^{\prime \prime}$, which is sufficient to cover the position with the FOV of the GLT.
An additional crucial advantage of SVOM follow-ups is the capability of detecting X-ray flashes (XRFs) and Xray-rich GRBs (XRRs) [58] and determining the prompt spectral peak energy $E_{\text {peak }}$. Because of the slightly higher energy range of Swift/BAT (15-150 keV), sample collections of XRFs and related rapid follow-ups have been entirely terminated. The $E_{\text {peak }}$ estimation of the Swift-detected GRBs (mainly XRRs and classical GRBs) has been provided by joint spectrum fittings of Swift/BAT and Suzaku/WAM [59]. Although spectral parameters of prompt emissions are adequately constrained by these joint fittings (e.g., [60-64]), the number of events is limited. This has caused a stagnation of the study of GRBs with prompt characterization. Because two of the prompt-emission-observing instruments onboard SVOM, ECLAIRs [65], and the Gamma Ray Monitor (GRM) [66] will cover the energy bands $4-150 \mathrm{keV}$ and $30-5000 \mathrm{keV}$, respectively, the $E_{\text {peak }}$ for most of SVOM-detected GRBs would be determined. In addition, numerous XRRs and XRFs would be detected with the $E_{\text {peak }}$ estimation through ECLIAS 


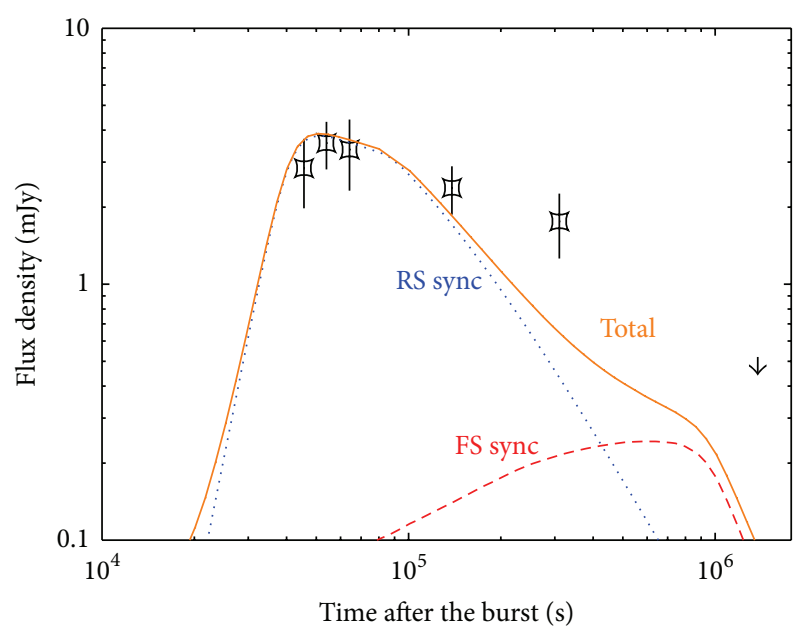

FIGURE 4: Submm light curve of GRB120326A with RS and FS model functions.

and GRM. According to the HETE-2 observations [58], the numbers of XRFs, XRRs, and GRBs were 16, 19, and 10, respectively. The number of softer events (XRFs/XRRs) was considerably higher than that of classical GRBs. Because the lower-energy coverage of HETE-2 (2-400 keV) [67] was similar to that of SVOM, a high volume of XRF and XRR samples with $E_{\text {peak }}$ measurements can be generated. This is likely to enhance the study of the origins of XRRs and XRFs by enabling the determination of their physical parameters. Hence, the GLT will be able to facilitate characterizing prompt and late-phase submm afterglows of all three types of bursts for the first time, providing crucial insights into the nature of XRFs and XRRs.

\section{Expected Science Cases}

On the basis of the summary of submm afterglow observations and the GLT project, we established the following three scientific goals.

5.1. Systematic Detection of Bright Submm Emissions. It is believed that RSs generate short bright optical flashes (e.g., [13]) and/or intense radio afterglows (e.g., [68]). According to the standard relativistic fireball model, RSs are expected to radiate emissions in the long wavelength bands (optical, infrared, and radio) by executing a synchrotron process in a particularly early afterglow phase (e.g., [69]).

Detecting this brief RS emission and measuring its magnitude would lead to constraints on several crucial parameters of the GRB ejecta, such as its initial Lorentz factor and magnetization [70]. Although RS emission has been detected in the optical wavelength in several GRBs, early optical observations for most GRBs have yielded no evidence of RS emission. The nondetection of RSs in optical bands could be an indicator of a magnetically dominated outflow. Another possible reason for the nondetection is that the typical RS synchrotron frequency is markedly below the optical band (e.g., [71]). Searching for RS emissions in the submm wavelength would test these possibilities. The comparison of early optical and submm temporal evolution would enable studying the composition of the GRB ejecta. If an RS component was regularly detected in GRBs of which the early optical light curve shows no evidence of RS emission, we would be able to claim that GRB ejecta are baryonic in nature. The detection of RS emissions in the submm band of most GRB would support the possibility that GRBs are baryonic flow.

One of the critical problems is that there has been no systematic submm observational study in the early afterglow phase of GRBs. As shown in Figure 1, the number of events that have been observed earlier than 1 day after bursts has remained at 16 for some time. The expected RS light curve for classical GRBs is fainter than $1 \mathrm{mJy}$ at 1 day after bursts and therefore undetectable using currently existing submm telescopes, excpet for ALMA. Figure 6 shows the expected RS light curves based on Kobayashi [69] and Kobayashi et al. [72] with various magnetic energy densities of RS $\epsilon_{B, \mathrm{RS}}$ and an initial Lorentz factor $\Gamma_{0}$ in comparison with the GLT sensitivity limit. In most of the cases shown in Figure 6, the RS component faded away before $1 \times 10^{5} \mathrm{~s}(\sim 1$ day). Hence, to detect and characterize RS components, rapid ( min scale) and continuous dense monitoring within 1 day is required. Although some of successful RS observations were made by SMA, CARMA, VLA, and others with their open use framework (e.g. [18, 42-44]), dedicated radio telescopes are strongly desired to make the systematic investigation. In addition, dense monitoring with the same wavelength is required to characterize the RS components, because sparse monitoring, even though rapid detection is included, failed to decode RS and FS components (e.g., [73]). Therefore, the use of the GLT would initiate the era of systematic submm follow-ups.

In Figure 6, cases of low initial Lorentz factors $(20,40)$ are provided showing XRRs and XRFs that are expected to be detected using the planned GRB satellite SVOM. The origin of the XRFs is not known. However, there are two major models, namely, (1) the failed GRBs or dirty fireball model (e.g., [74]) and (2) the off-axis jet model [75]. According to the dirty fireball model, low-inital-Lorentz-factor $\left(\Gamma_{0} \ll\right.$ $100)$, GRBs produce a lower spectral peak energy $E_{\text {peak }}$ in the prompt phase because of $E_{\text {peak }} \propto \Gamma_{0}^{4}$ dependence, and it is therefore natural to attribute this energy to XRRs and XRFs. The low Lorentz factors substantially delay the RS peak (Figure 6(b)). For the latter model, it is assumed that the viewing angle is considerably larger than the collimation angle of the jet, and the high ratio of X-ray to $\gamma$-ray fluence is caused by a relativistic beaming factor when a GRB is observed through off-axis direction. The key observable feature is the achromatic brightening optical afterglow light curves, of which the peak time depends on the viewing angle $[76,77]$. Hence, identifying a delayed RS peak through rapid GLT monitoring and the prompt spectral characterization of SVOM will confirm and identify the origin of XRFs and XRRs. 


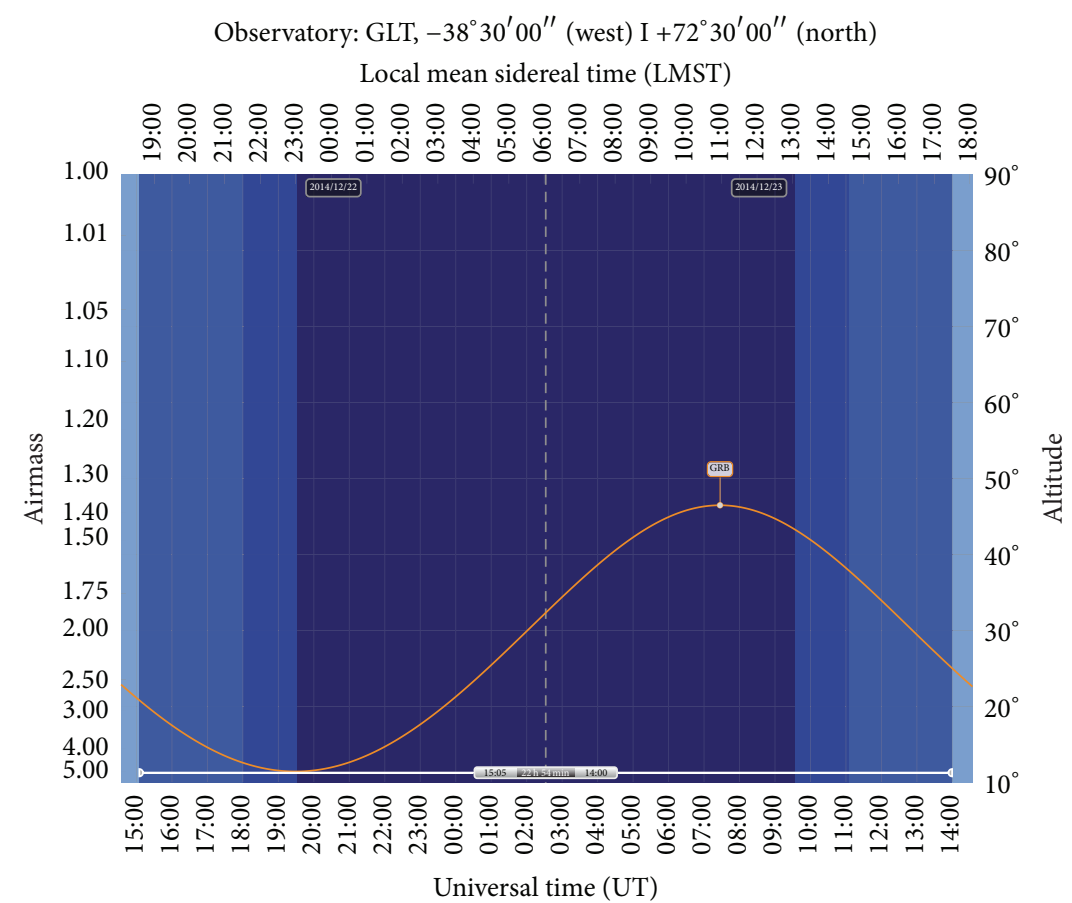

FIGURE 5: An example of visibility plot in winter for the GRB that declination is 29 deg. Hatchs indicate the sun altitude lower than -18 (dark blue), -12 (blue), -6 (dark cyan), and 0 (cyan) deg., respectively.

5.2. Characterization of Forward and Reverse Shock Emissions. The spectral characteristics of FS and RS synchrotron emissions are related as follows: $v_{m, \mathrm{FS}} \sim \mathscr{R}_{B}^{1 / 2} \mathscr{R}_{M}^{2} \nu_{m, \mathrm{RS}}$, and $F_{\text {max }, \mathrm{RS}} \sim \mathscr{R}_{M} \mathscr{R}_{B}^{-1 / 2} F_{\text {max,FS }}$ (e.g., [70]), where $\mathscr{R}_{M}\left(=\Gamma_{d}^{2} / \Gamma_{0}\right)$ and $\mathscr{R}_{B}\left(=\epsilon_{B, \mathrm{FS}} / \epsilon_{B, \mathrm{RS}}\right)$ denote the mass and magnetization ratio parameters, respectively. Here, $\Gamma_{d}, \epsilon_{B, \mathrm{FS}}$, and $\epsilon_{B, \mathrm{RS}}$ are the Lorentz factors at the deceleration time, the fractions of magnetic energy of RS and FS, respectively. RS emission is typically expected to be considerably brighter ( 100 times) than FS emission as shown in Figure 7. Therefore, the emission from RSs is sensitive to the properties of the fireball, and the broadband spectrum and light curve evolutions of FS/RS can provide critical clues to understanding GRBs.

Regarding FSs, afterglows can be described by synchrotron emissions from a decelerating relativistic shell that collides with an external medium. According to the FS synchrotron model, both the spectrum and light curve consist of several power-law segments with related indices (e.g., [78, 79]). The broadband spectrum is characterized according to the synchrotron peak frequency $v_{m, \mathrm{FS}}$ and the peak spectrum flux density $F_{\max , F s}$. The peak spectrum flux is expected to occur at low frequencies (from X-ray to radio) over time (from minutes to several weeks) as $v_{m, \mathrm{FS}} \propto t^{-3 / 2}$. The peak spectrum flux density $F_{\text {max,FS }}$ is predicted to remain constant in the circumburst model, whereas it decreases as $F_{\text {max } \mathrm{FS}} \propto t^{-1 / 2}$ in the wind model. Therefore, determining the characterizing frequencies and peak fluxes by using temporal and spectrum observations provides direct evidence of the FS/RS shock synchrotron model and typical (or average) physical conditions of a fireball.
Snapshots of the broadband spectrum and continuous monitoring of light curves in the submm bands are essential to characterizing the radiation of afterglow by decoding each component. Figure 8 shows the expected light curve in the $230 \mathrm{GHz}$ band at $z=0.3,0.5$, and 0.7. We fix the rest of parameters as explosion energy $E=3 \times 10^{52} \mathrm{erg}$, circumburst number density $n=1 \mathrm{~cm}^{-3}$, the electron spectral index $p=2.1$, the electron energy density $\epsilon_{e}=0.3$, and the magnetic energy density of FS $\epsilon_{B, F S}=0.01$. The brightening caused by the passing of the synchrotron peak frequency $v_{m}$ can be detected to determine the FS component in light curves. The GLT has also enough sensitivities to detect FS component for nearby $(z \leqslant 0.7)$ events and the monitoring determines their $v_{m, \mathrm{FS}}$. The expected $v_{m, \mathrm{FS}}$ passing through time across the $230 \mathrm{GHz}$ band is several $\times 10^{5} \mathrm{~s}$ (Figure 8). Therefore, the submm band is suitable for decoding both RS and FS components. Some of closure relations for FS and RS [79] will also constrain components even if the light curve or spectrum observations are sparsely performed. For X-ray and OIR cases, the expected timescale is between several minutes and $\sim 2$ hours after the burst. In this time range, detecting the peak frequency directly is difficult because several additional components (e.g., long-lasting prompt emission, X-ray flares, etc.) characterize this phase. For the MIR case, observations fully rely on the satellite-based instruments. In this case, timely follow-ups are difficult because of operation confinement and limitation of number of resources. Furthermore, the slow temporal evolution in the radio band makes it difficult to obtain simultaneous optical and X-ray segments. This creates uncertainty regarding whether we observe the same synchrotron components or not. Hence, the GLT will 


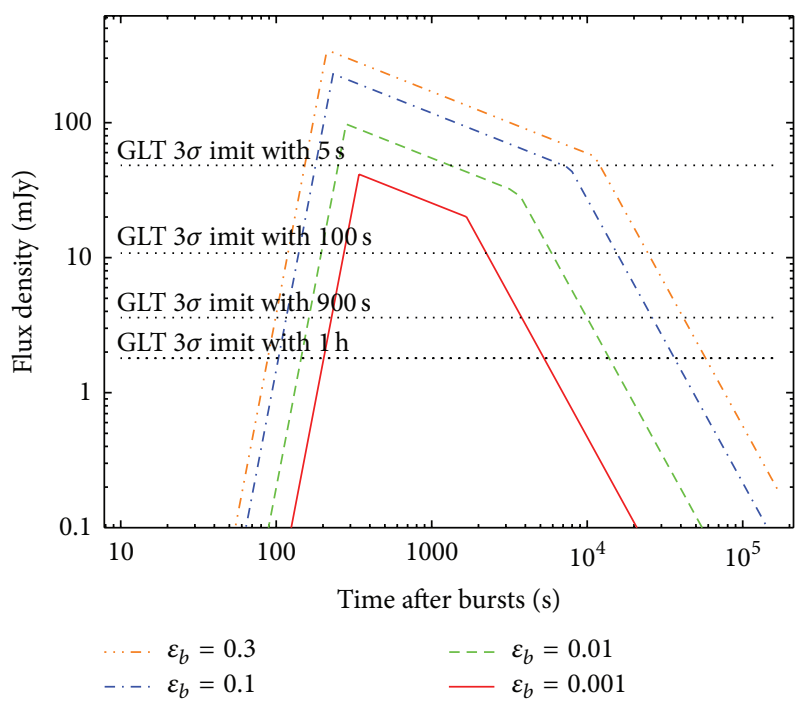

(a)

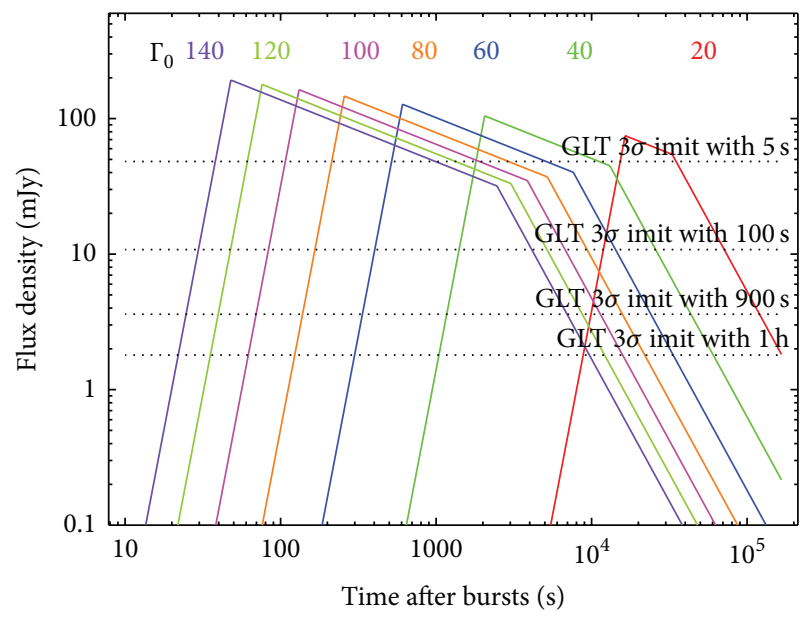

(b)

Figure 6: (a) Expected RS light curves in the $230 \mathrm{GHz}$ band with various $\epsilon_{B, \mathrm{RS}}(0.001,0.01,0.1$, and 0.3$)$. Other physical parameters are fixed as $z=1, E=3 \times 10^{52}, \Gamma=80, n=1, \epsilon_{e}=0.3$, and $\epsilon_{B, \mathrm{FS}}=0.01$. (b) Expected RS light curves in the $230 \mathrm{GHz}$ band with various initial Lorentz factor $\Gamma_{0}$. Other physical parameters are $z=1, E=3 \times 10^{52}$, $n=1, \epsilon_{e}=0.3, \epsilon_{B, \mathrm{RS}}=0.03$, and $\epsilon_{B, \mathrm{FS}}=0.01$. The expected GLT 3- $\sigma$ limits with $900 \mathrm{~s}$ and $1 \mathrm{~h}$ exposure are indicated with dotted lines in both panels.

provide unique results for nearby events $(z \lesssim 0.7)$ by facilitating continuous monitoring.

Optical monitoring combined with the GLT will be required. As we describe above, RS components will be caught by submm observations. For characterizing FS components, multicolor optical monitoring is suitable because the temporal evolution and spectrum of optical afterglow around 1 day after bursts are well consistent with the expectation of the FS model. Figure 3 shows one of the most appropriate examples of the earliest submm afterglow detection procedures performed using the SMA and related optical monitoring [18]. Because of the rapid submm monitoring, the FS and

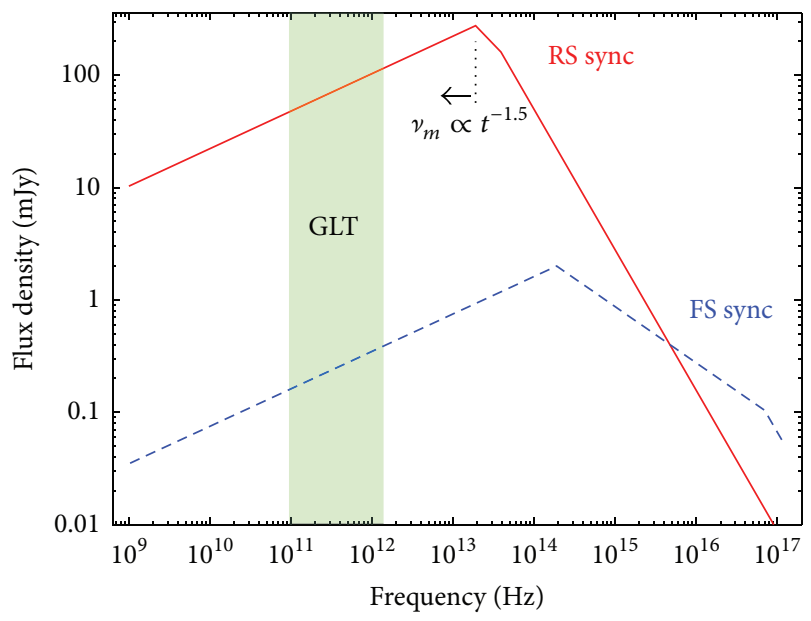

FIgURE 7: Expected spectrum at the deceleration time with $z=1$, $E=1 \times 10^{52}, \Gamma=80, n=1, \epsilon_{e}=0.3, \epsilon_{B, \mathrm{RS}}=0.03$, and $\epsilon_{B, \mathrm{FS}}=0.01$. Synchrotron radiations from reverse and forward shock are indicated by red solid and blue dashed lines, respectively. A green hatched box indicates the frequency coverage of GLT.

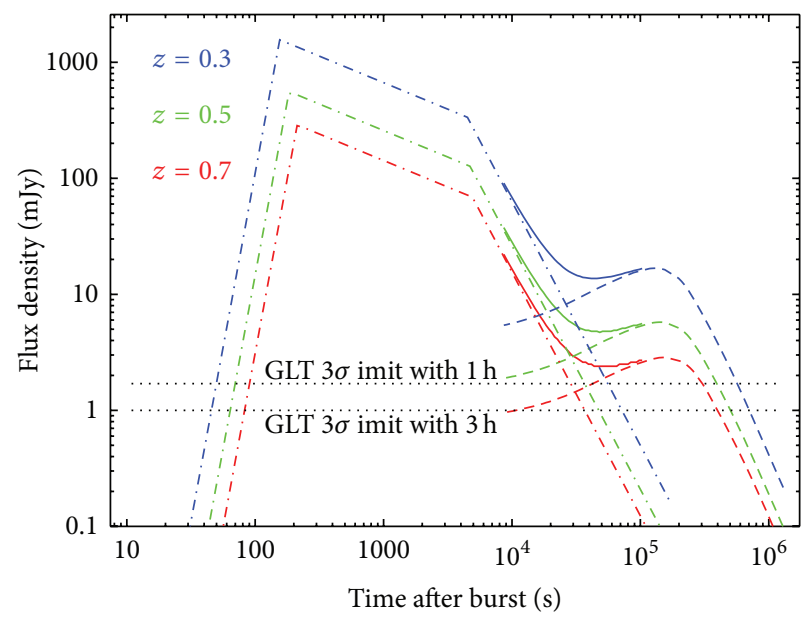

FIgURE 8: Expected RS (dashed-dot) and FS (dashed) light curves in the $230 \mathrm{GHz}$ band at $z=0.3,0.5$, and 0.7 with $E=3 \times 10^{52} \mathrm{erg}$, $n=1 \mathrm{~cm}^{-3}, p=2.1, \epsilon_{e}=0.3$, and $\epsilon_{B, \mathrm{FS}}=0.01$. Solid lines indicate the total of RS and FS. GLT has also enough sensitivity to characterize the FS components for nearby $(z \lesssim 0.7)$ GRBs. The expected GLT 3- $\sigma$ limits with $1 \mathrm{~h}$ and $3 \mathrm{~h}$ exposure are indicated with dotted lines.

RS components were separated. Conducting multifrequency monitoring by using the GLT requires rapid optical followups, which will be conducted using our own optical network EAFON [80-82] and other ground-based optical telescopes, as numerous observations have been conducted.

5.3. Discovering of First-Star Explosions by Using GRBs. The findings regarding a high-redshift GRB at $z=8.2$ [1] indicated the possibility of using GRBs to probe the processes and environments of star formation as far back in time as the earliest cosmic epochs. Numerous theoretical models (e.g., [83-85]) show that some POP-III stars generate GRBs as 


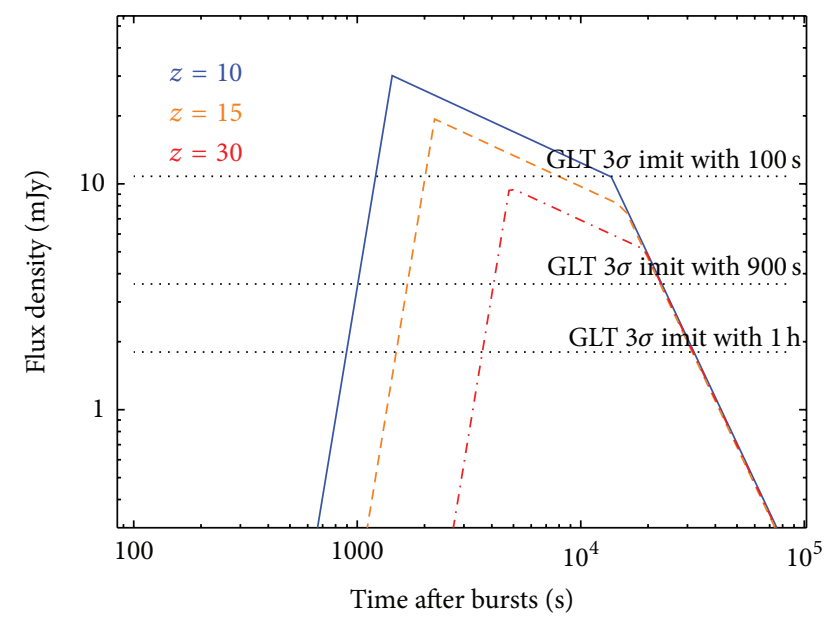

FigURE 9: Expected light curves of GRB afterglows at $z=10,15$, and 30 at $230 \mathrm{GHz}$. Other physical parameters are fixed as $E=1 \times 10^{53}$, $\Gamma=80, n=1, \epsilon_{e}=0.3, \epsilon_{B, \mathrm{RS}}=0.03$, and $\epsilon_{B, \mathrm{FS}}=0.01$. The expected GLT 3- $\sigma$ limits with $900 \mathrm{~s}$ and $1 \mathrm{~h}$ exposure are indicated with dotted lines. GLT with rapid follow-ups has sufficient potential to detect these higher- $z$ events.

an end product. Hence, detecting the signals of high- $z$ GRBs has been a recent prominent objective in modern astrophysics.

One of the prospective methods of identifying POP-III GRBs is to detect RS components in the submm bands. Unlike OIR observations, submm observations provide clean measurements of the source intensity, unaffected by extinction. Because of the intense luminosity of the RSs, it is expected that the radiation from high- $z$ GRBs $(z>10-30)$ can be observed if the GLT is used with the rapid response system. Inoue et al. [86] showed that the RS component of POP-III GRBs at $z=15$ and 30 in the $300 \mathrm{GHz}$ band is substantially brighter than $1 \mathrm{mJy}$, and these bright RS components will be detectable by using the GLT. In addition, we simulated the expected RS light curves at $z=10,15$, and 30 based on Kobayashi [69] and Kobayashi et al. [72]. For this calculation, we assumed that $E=1 \times 10^{53}$ because the progenitor stars might be considerably more massive than nearby events (e.g., [83]). Other physical parameters are fixed as $\Gamma=80, n=$ $1, \epsilon_{e}=0.3, \epsilon_{B, \mathrm{RS}}=0.03$, and $\epsilon_{B, \mathrm{FS}}=0.01$. As shown in Figure 9, the GLT has great potential to detect the high$z$ GRBs even those at $z=30$ with the rapid responding, shorter exposure cycle, and continuous dense monitoring. These initial detections of the GLT in the early phase will provide the opportunity to conduct additional follow-ups using 30-m class telescopes such as the TMT. Because these large telescopes typically enable conducting follow-ups for a limited number of events, the target selections of the GLT observations will be critical.

The event rate of high- $z$ GRBs, which may be connected to the star-formation rate in the early universe, is not known. Wanderman and Piran [87] estimated that the event rate of high- $z(z>7)$ GRBs might be $\sim 10$ events per year per steradian on the basis of limited 100 Swift-detected long GRBs with known redshift and measured peak flux. Their estimation showed that Swift/BAT exhibited substantially high redshift fractions $(\sim 3.4 \%$ at $z>7)$, whereas Swift and related follow-ups detected only a few $z>7$ events. Hence, uncertainty exists regarding the number of higher- $z$ events that Swift has detected; thus, the frequency of such events is not thoroughly understood because appropriate follow-ups in long wavelength (e.g., IR, submm) have not been conducted. Actually, $z \sim 9$ GRB candidate [88] was also detected through Swift. Therefore, a continuous effort is required in this field of research, despite a success rate that is typically low. In addition, Wanderman and Piran [87] expected that SVOM will detect $0.1-7$ events per year at $z>$ 10. To detect these events, rapid follow-up coordination with submm instruments will be crucial, because it is impossible to identify higher- $z$ candidates within hours from bursts with limited observational information. Therefore, the installing of a rapid responding system in the GLT will enable us to perform high- $z$ GRB observations.

\section{Summary}

We briefly summarized the current achievements of submm follow-up observations of GRBs. Although submm afterglow observations are critical to understanding the nature of GRBs (e.g., GRB030329 and GRB120326A), the number of successful observations has been limited. This is because of the lack of dedicated submm telescopes that has made it difficult to perform rapid follow-up.

Furthermore, we introduced the single-dish mode of GLT. The development is ongoing and the expected first light of the GLT will be made in 2017/18. The first light instrument will be the VLBI receivers at 86,230 , and $345 \mathrm{GHz}$. The expected sensitivities of the receivers are 36 and $88 \mathrm{mJy}$ in $1 \mathrm{~s}$ integration at 230 and $345 \mathrm{GHz}$, respectively. The GLT enables rapid and continuous submm monitoring of GRB submm counterparts in the prompt phase.

According to the aforementioned situations and expected capabilities of the GLT, we established the following three key scientific goals regarding GRB studies: (1) systematic detection of bright submm emissions originating from RS in the early afterglow phase by conducting rapid follow-ups, (2) characterization of FS and RS emissions by capturing their peak flux and frequencies through continuous monitoring, and (3) detection of the first star explosions as a result of GRBs at a high redshift through systematic rapid follow-ups.

Detecting RS emissions and monitoring light curves in the submm band could lead to constraints on several crucial parameters of the GRB ejecta, such as the initial Lorentz factor and magnetization. We calculated the expected RS light curves by using various initial Lorentz factors $\Gamma_{0}$ and the magnetic energy densities of RS $\epsilon_{B, \mathrm{RS}}$ and showed that these light curves could be characterized through rapid follow-ups of the GLT. Determining the origins of XRRs and XRFs will also be a major focus of the GLT together with the SVOM mission.

In addition to characterizing RS components, the GLT will be able to detect FS components of nearby $(z \lesssim 0.7)$ GRBs. Because the spectral characteristics of the FS and RS 
synchrotron emissions are related, characterizing both FS and RS components provides critical insights into GRBs. We generated an expected submm light curve at $z=0.3,0.5$, and 0.7 and showed that the GLT can separate RS and FS components through long-term and continuous monitoring, because RS and FS components dominate earlier than $\sim 4 \times$ $10^{4} \mathrm{~s}$ and later than $\sim 10^{5} \mathrm{~s}$, respectively.

These two RS science topics will enhance the GRB studies as the probe of a high- $z$ universe. Because of the existence of RS and its extreme luminosity, it is expected that the radiation from high- $z$ GRB $(z>10-30)$ can be observed if the GLT is used with a rapid (hours scale) response system. We simulated the expected RS light curves at $z=10,15$, and 30. These light curves showed that the GLT has sufficient sensitivity to detect and characterize these events. The future SVOM mission may provide the capability to detect GRBs at $z>10$; the establishment of close coordination using longer wavelength (e.g., infrared, submm) instruments will be crucial. Because the rapid identification of counterparts at long wavelengths is crucial for conducting additional further follow-ups using 30-m class telescopes such as the TMT will be used. Therefore, the GLT could play a crucial role in detecting high $-z$ GRBs.

\section{Conflict of Interests}

The authors declare that there is no conflict of interests regarding the publication of this paper.

\section{Acknowledgments}

The Greenland Telescope (GLT) Project is a collaborative project between Academia Sinica Institute of Astronomy and Astrophysics, Smithsonian Astrophysical Observatory, MIT Haystack Observatory, and National Radio Astronomy Observatory. The authors would like to thank Shiho Kobayashi for the useful comments. The authors would also like to thank all members of the GLT single-dish science team organized at ASIAA. This work is partly supported by the Ministry of Science and Technology of Taiwan Grants MOST 103-2112-M-008-021- (Yuji Urata), 103-2112-M-001-038-MY2 (Keiichi Asada), and 102-2119-M-001-006-MY3 (Hiroyuki Hirashita).

\section{References}

[1] N. R. Tanvir, D. B. Fox, A. J. Levan et al., "A $\gamma$-ray burst at a redshift of $\mathrm{z} \approx 8.2$," Nature, vol. 461, no. 7268, pp. 1254-1257, 2009.

[2] J. S. Bloom, D. A. Perley, W. Li et al., "Observations of the nakedeye GRB 080319B: implications of nature's brightest explosion," Astrophysical Journal, vol. 691, no. 1, pp. 723-737, 2009.

[3] K. Z. Stanek, T. Matheson, P. M. Garnavich et al., "Spectroscopic discovery of the supernova $2003 \mathrm{dh}$ associated with GRB 030329," Astrophysical Journal Letters, vol. 591, no. 1, pp. L17L20, 2003.

[4] P. Mészáros and M. J. Rees, "Optical and long-wavelength afterglow from gamma-ray bursts," The Astrophysical Journal, vol. 476, no. 1, pp. 232-237, 1997.
[5] N. Gehrels, G. Chincarini, P. Giommi et al., “The Swift gammaray burst mission," The Astrophysical Journal, vol. 611, no. 2, p. 1005, 2004.

[6] I. A. Steele, "The Liverpool robotic telescope," New Astronomy Reviews, vol. 45, no. 1-2, pp. 45-47, 2001.

[7] C. W. Akerlof, R. L. Kehoe, T. A. McKay et al., "The ROTSEIII robotic telescope system," Publications of the Astronomical Society of the Pacific, vol. 115, no. 803, pp. 132-140, 2003.

[8] W. T. Vestrand, K. N. Borozdin, S. P. Brumby et al., "The RAPTOR experiment: a system for monitoring the optical sky in real time," in 2nd Advanced Global Communications Technologies for Astronomy, vol. 4845 of Proceedings of SPIE, pp. 126-136, Waikoloa, Hawaii, USA, November 2002.

[9] A. Burd, M. Cwiok, H. Czyrkowski et al., "Pi of the sky-all-sky, real-time search for fast optical transients," New Astronomy, vol. 10, no. 5, pp. 409-416, 2005.

[10] A. Klotz, M. Boër, J. L. Atteia, and B. Gendre, "Early optical observations of gamma-ray bursts by the TAROT telescopes: period 2001-2008," Astronomical Journal, vol. 137, no. 5, pp. 4100-4108, 2009.

[11] A. Ferrero, L. Hanlon, R. Felletti et al., "The photometry pipeline of the watcher robotic telescope," Advances in Astronomy, vol. 2010, Article ID 715237, 5 pages, 2010.

[12] Y. Urata, M. S. Tashiro, T. Tamagawa et al., "WIDGET: system performance and GRB prompt optical observations," Publications of the Astronomical Society of Japan, vol. 63, no. 1, pp. 137146, 2011.

[13] C. Akerlof, R. Balsano, S. Barthelmy et al., "Observation of contemporaneous optical radiation from a $\gamma$-ray burst," Nature, vol. 398, no. 6726, pp. 400-402, 1999.

[14] W. T. Vestrand, P. R. Wozniak, J. A. Wren et al., "A link between prompt optical and prompt $\gamma$-ray emission in $\gamma$-ray bursts," Nature, vol. 435, no. 7039, pp. 178-180, 2005.

[15] W. T. Vestrand, J. A. Wren, P. R. Wozniak et al., "Energy input and response from prompt and early optical afterglow emission in $\gamma$-ray bursts," Nature, vol. 442, no. 7099, pp. 172-175, 2006.

[16] J. L. Racusin, S. V. Karpov, M. Sokolowski et al., "Broadband observations of the naked-eye $\gamma$-ray burst GRB 080319B," Nature, vol. 455, pp. 183-188, 2008.

[17] P. Chandra and D. A. Frail, "A radio-selected sample of gammaray burst afterglows," The Astrophysical Journal, vol. 746, no. 2, p. 156, 2012.

[18] Y. Urata, K. Huang, S. Takahashi et al., "Synchrotron self-inverse compton radiation from reverse shock on GRB 120326A," The Astrophysical Journal, vol. 789, p. 146, 2014.

[19] D. A. Smith, E. S. Rykoff, C. W. Akerlof et al., "ROTSEIII observations of the early afterglow from GRB 030329," Astrophysical Journal Letters, vol. 596, no. 2, pp. L151-L154, 2003.

[20] A. Tiengo, S. Mereghetti, G. Ghisellini, E. Rossi, G. Ghirlanda, and N. Schartel, “The X-ray afterglow of GRB 030329," Astronomy and Astrophysics, vol. 409, no. 3, pp. 983-987, 2003.

[21] S. Klose, E. Palazzi, N. Masetti et al., "Prospects for multiwavelength polarization observations of GRB afterglows and the case GRB 030329," Astronomy \& Astrophysics, vol. 420, no. 3, pp. 899-903, 2004.

[22] G. Kosugi, Y. Mizumoto, N. Kawai et al., "Spectral evolution of the GRB 030329 afterglow: detection of the supernova nebular phase emissions," Publications of the Astronomical Society of Japan, vol. 56, no. 1, pp. 61-68, 2004.

[23] Y. Urata, T. Miyata, S. Nishiura et al., "Early $(<0.3$ days $) R-$ band light curve of the optical afterglow of GRB 030329," Astrophysical Journal Letters, vol. 601, no. 1, pp. L17-L19, 2004. 
[24] A. Tiengo, S. Mereghetti, G. Ghisellini, F. Tavecchio, and G. Ghirlanda, "Late evolution of the X-ray afterglow of GRB 030329," Astronomy and Astrophysics, vol. 423, no. 3, pp. 861865, 2004.

[25] N. Kuno, N. Sato, H. Nakanishi et al., "Radio observations of the afterglow of GRB 030329," Publications of the Astronomical Society of Japan, vol. 56, pp. L1-L4, 2004.

[26] V. Šimon, R. Hudec, and G. Pizzichini, "The color evolution of the optical afterglow of GRB 030329 and the implications for the underlying supernova SN 2003dh," Astronomy and Astrophysics, vol. 427, no. 3, pp. 901-905, 2004.

[27] D. A. Frail, A. M. Soderberg, S. R. Kulkarni et al., "Accurate calorimetry of GRB 030329," The Astrophysical Journal, vol. 619, no. 2, pp. 994-998, 2005.

[28] K. Kohno, T. Tosaki, T. Okuda et al., "Nobeyama millimeter array observations of GRB 030329: a decay of afterglow with bumps and molecular gas in the host galaxy," Publications of the Astronomical Society of Japan, vol. 57, no. 1, pp. 147-153, 2005.

[29] I. A. Smith, R. P. J. Tilanus, N. Tanvir et al., "SCUBA submillimeter observations of gamma-ray bursts: III. GRB 030329: the brightest sub-millimeter afterglow to date," Astronomy and Astrophysics, vol. 439, no. 3, pp. 981-986, 2005.

[30] J. Gorosabel, D. Pérez-Ramírez, J. Sollerman et al., “The GRB 030329 host: a blue low metallicity subluminous galaxy with intense star formation," Astronomy \& Astrophysics, vol. 444, no. 3, pp. 711-721, 2005.

[31] J. Van Der Horst, A. Kamble, L. Resmi et al., "Detailed study of the GRB 030329 radio afterglow deep into the non-relativistic phase," Astronomy and Astrophysics, vol. 480, no. 1, pp. 35-43, 2008.

[32] K. Sheth, D. A. Frail, S. White et al., "Millimeter observations of GRB 030329: continued evidence for a two-component jet," Astrophysical Journal Letters, vol. 595, no. 1, pp. L33-L36, 2003.

[33] L. Resmi, C. H. Ishwara-Chandra, A. J. Castro-Tirado et al., "Radio, millimeter and optical monitoring of GRB 030329 afterglow: constraining the double jet model," Astronomy and Astrophysics, vol. 440, no. 2, pp. 477-485, 2005.

[34] P. A. Price, D. W. Fox, S. R. Kulkarni et al., "The bright optical afterglow of the nearby $\gamma$-ray burst of 29 March 2003," Nature, vol. 423, no. 6942, pp. 844-847, 2003.

[35] E. Berger, S. R. Kulkarni, G. Pooley et al., "A common origin for cosmic explosions inferred from calorimetry of GRB030329," Nature, vol. 426, no. 6963, pp. 154-157, 2003.

[36] P. T. P. Ho, J. M. Moran, and K. Y. Lo, “The submillimeter array," The Astrophysical Journal, vol. 616, no. 1, article L1, 2004.

[37] A. de Ugarte Postigo, A. Lundgren, S. Martín et al., "Pre-ALMA observations of GRBs in the $\mathrm{mm} /$ submm range," Astronomy \& Astrophysics, vol. 538, article A44, 23 pages, 2012.

[38] F. E. Marshall, L. A. Antonelli, D. N. Burrows et al., "The late peaking afterglow of GRB 100418A," The Astrophysical Journal, vol. 727, article 132, 2011.

[39] A. Panaitescu, P. Mészáros, D. Burrows et al., "Evidence for chromatic X-ray light-curve breaks in Swift gamma-ray burst afterglows and their theoretical implications," Monthly Notices of the Royal Astronomical Society, vol. 369, no. 4, pp. 2059-2064, 2006.

[40] K. Y. Huang, Y. Urata, P. H. Kuo et al., "Multicolor shallow decay and chromatic breaks in the GRB 050319 optical afterglow," The Astrophysical Journal Letters, vol. 654, no. 1, pp. L25-L28, 2007.
[41] Y. Urata, R. Yamazaki, T. Sakamoto et al., "Testing the externalshock model of gamma-ray bursts using the late-time simultaneous optical and X-ray afterglows," The Astrophysical Journal Letters, vol. 668, no. 2, pp. L95-L98, 2007.

[42] T. Laskar, E. Berger, B. A. Zauderer et al., "A reverse shock in GRB 130427A," Astrophysical Journal, vol. 776, no. 2, article 119, 2013.

[43] D. A. Perley, S. B. Cenko, A. Corsi et al., "The afterglow of GRB 130427A from 1 to 1016 GHz," The Astrophysical Journal, vol. 781, no. 1, article 37, 2014.

[44] A. J. van der Horst, Z. Paragi, A. G. de Bruyn et al., "A comprehensive radio view of the extremely bright gammaray burst 130427A," Monthly Notices of the Royal Astronomical Society, vol. 444, no. 4, pp. 3151-3163, 2014.

[45] I. A. Smith, R. P. J. Tilanus, N. R. Tanvir, and D. A. Frail, "GRB 120404A: JCMT SCUBA-2 sub-mm observation," GRB Coordinates Network, vol. 13233, p. 1, 2012.

[46] I. A. Smith, R. P. J. Tilanus, N. R. Tanvir, and D. A. Frail, "GRB 130131A: JCMT SCUBA-2 sub-mm observations," GRB Coordinates Network, vol. 13259, p. 1, 2012.

[47] I. A. Smith, R. P. J. Tilanus, N. R. Tanvir, and D. A. Frail, "GRB 120724A: JCMT SCUBA-2 sub-mm observation," GRB Coordinates Network, vol. 13519, p. 1, 2012.

[48] I. A. Smith, R. P. J. Tilanus, N. R. Tanvir, and D. A. Frail, "GRB 120729A: JCMT SCUBA-2 sub-mm observation," GRB Coordinates Network, vol. 13554, p. 1, 2012.

[49] I. A. Smith, R. P. J. Tilanus, N. R. Tanvir, and D. A. Frail, "GRB 120422A: JCMT SCUBA-2 sub-mm observation," GRB Coordinates Network, vol. 14281, p. 1, 2013.

[50] I. A. Smith, R. P. J. Tilanus, N. R. Tanvir, and D. A. Frail, "GRB 130831A: JCMT SCUBA-2 sub-mm observation," GRB Coordinates Network, vol. 15174, p. 1, 2013.

[51] M. Inoue, J. C. Algaba-Marcos, K. Asada et al., "Greenland telescope project: direct confirmation of black hole with submillimeter VLBI," Radio Science, vol. 49, no. 7, pp. 564-571, 2014.

[52] H. Hirashita, P. M. Koch, S. Matsushita et al., "First-generation science cases for ground-based Terahertz telescopes," Publications of the Astronomical Society of the Pacific. Submitted.

[53] P. L. Martin-Cocher, K. Asada, S. Matsushita, M.-T. Chen, P. T. P. Ho, and C.-P. Chen, " $225 \mathrm{GHz}$ opacity measurements at Summit camp, Greenland, for the GreenLand Telescope (GLT) site testing," in 5th Ground-based and Airborne Instrumentation for Astronomy, vol. 9147 of Proceedings of SPIE, p. 91473N, Montréal, Canada, July 2014.

[54] P. K. Grimes, K. Asada, R. Blundell et al., "Instrumentation for single-dish observations with The Greenland Telescope," in Millimeter, Submillimeter, and Far-Infrared Detectors and Instrumentation for Astronomy VII, vol. 9153 of Proceedings of SPIE, Montreal, Canada, June 2014.

[55] O. Godet, J. Paul, J. Y. Wei et al., "The Chinese-French SVOM mission: studying the brightest astronomical explosions," in Space Telescopes and Instrumentation 2012: Ultraviolet to Gamma Ray, Proceedings of SPIE, 84431O, September 2012.

[56] T. Sakamoto and N. Gehrels, "Review the anti-sun pointing operation of Swift," in Proceedings of the Swift Mission Conference: Celebrating 5 Years, State College, Pa, USA, November 2009.

[57] D. Götz, J. Osborne, B. Cordier et al., "The microchannel Xray telescope for the gamma-ray burst mission SVOM," in Space Telescopes and Instrumentation 2014: Ultraviolet to Gamma Ray, vol. 9144 of Proceedings of SPIE, p. 914423, Montréal, Canada, July 2014. 
[58] T. Sakamoto, D. Q. Lamb, N. Kawai et al., "Global characteristics of X-ray flashes and X-ray-rich gamma-ray bursts observed by HETE-2," The Astrophysical Journal, vol. 629, p. 311, 2005.

[59] K. Yamaoka, A. Endo, T. Enoto et al., "Design and in-orbit performance of the Suzaku wide-band all-sky monitor," Publications of the Astronomical Society of Japan, vol. 61, supplement 1, pp. S35-S53, 2009.

[60] R. L. C. Starling, P. T. O’Brien, R. Willingale et al., "Swift captures the spectrally evolving prompt emission of GRB 070616," Monthly Notices of the Royal Astronomical Society, vol. 384, no. 2, pp. 504-514, 2008.

[61] H. A. Krimm, K. Yamaoka, S. Sugita et al., "Testing the EpeakE iso relation for GRBs detected by Swift and Suzaku-WAM," Astrophysical Journal Letters, vol. 704, no. 2, pp. 1405-1432, 2009.

[62] S. Sugita, K. Yamaoka, M. Ohno et al., "Suzaku-WAM, KonusWind, and Swift-BAT observations of prompt emission of the high-redshift GRB 050904," Publications of the Astronomical Society of Japan, vol. 61, no. 3, pp. 521-527, 2009.

[63] Y. Urata, K. Huang, M. Im et al., "Swift GRB GRB071010B: outlier of the $E_{\text {peak }}^{s r c}-E_{\gamma}$ and $E_{\text {iso }}-E_{\text {peak }}^{s r c}-t_{\text {jet }}^{s r c}$ correlations," The Astrophysical Journal, vol. 706, no. 7, p. L183, 2009.

[64] Y. Urata, K. Huang, K. Yamaoka, P. P. Tsai, and M. S. Tashiro, "Energetic Fermi/LAT GRB100414A: energetic and correlations," Astrophysical Journal Letters, vol. 748, no. 1, article L4, 2012.

[65] O. Godet, G. Nasser, J. Atteia et al., “The X-/gamma-ray camera ECLAIRs for the gamma-ray burst mission SVOM," in Space Telescopes and Instrumentation 2014: Ultraviolet to Gamma Ray, vol. 9144 of Proceedings of SPIE, p. 914424, Montréal, Canada, July 2014.

[66] Y. Dong, B. Wu, Y. Li, Y. Zhang, and S. Zhang, "SVOM gamma ray monitor," Science China Physics, Mechanics and Astronomy, vol. 53, no. 1, supplement, pp. 40-42, 2010.

[67] Y. Shirasaki, N. Kawai, A. Yoshida et al., "Design and performance of the wide-field X-ray monitor on board the highenergy transient explorer 2," Publications of the Astronomical Society of Japan, vol. 55, pp. 1033-1049, 2003.

[68] S. R. Kulkarni, D. A. Frail, R. Sari et al., "Discovery of a radio flare from GRB 990123," Astrophysical Journal Letters, vol. 522, no. 2, pp. L97-L100, 1999.

[69] S. Kobayashi, "Light curves of gamma-ray burst optical flashes," The Astrophysical Journal, vol. 545, no. 2, pp. 807-812, 2000.

[70] B. Zhang, S. Kobayashi, and P. Mészáros, "Gamma-ray burst early optical afterglows: implications for the initial Lorentz factor and the central engine," The Astrophysical Journal, vol. 595, no. 2, pp. 950-954, 2003.

[71] A. Melandri, S. Kobayashi, C. G. Mundell et al., "GRB 090313 and the origin of optical peaks in gamma-ray burst light curves: implications for Lorentz factors and radio flares," Astrophysical Journal Letters, vol. 723, no. 2, pp. 1331-1342, 2010.

[72] S. Kobayashi, B. Zhang, P. Mészáros, and D. Burrows, "Inverse compton X-ray flare from gamma-ray burst reverse shock," The Astrophysical Journal, vol. 655, no. 1, p. 391, 2007.

[73] P. Veres, A. Corsi, D. A. Frail, S. B. Cenko, and D. A. Perley, "Early-time VLA observations and broad-band afterglow analysis of the Fermi-LAT detected GRB 130907A," http://arxiv.org/abs/1411.7368.

[74] Y. F. Huang, Z. G. Dai, and T. Lu, "Failed gamma-ray bursts and orphan afterglows," Monthly Notices of the Royal Astronomical Society, vol. 332, no. 3, pp. 735-740, 2002.
[75] R. Yamazaki, K. Ioka, and T. Nakamura, "X-ray flashes from offaxis gamma-ray bursts," Astrophysical Journal Letters, vol. 571, no. 1, pp. L31-L35, 2002.

[76] E. Nakar and T. Piran, "On-axis orphan afterglows," New Astronomy, vol. 8, no. 2, pp. 141-153, 2003.

[77] Y. Urata, K. Huang, R. Yamazaki, and T. Sakamoto, "Extremely soft X-ray flash as the indicator of off-axis orphan GRB afterglow," The Astrophysical Journal. In press.

[78] R. Sari, T. Piran, and J. P. Halpern, "Jets in gamma-ray bursts," Astrophysical Journal Letters, vol. 519, no. 1, pp. L17-L20, 1999.

[79] H. Gao, W.-H. Lei, Y.-C. Zou, X.-F. Wu, and B. Zhang, "A complete reference of the analytical synchrotron external shock models of gamma-ray bursts," New Astronomy Reviews, vol. 57, no. 6, pp. 141-190, 2013.

[80] K. Y. Huang, Y. Urata, A. V. Filippenko et al., "Optical afterglow observations of the unusual short-duration gamma-ray burst GRB 040924," Astrophysical Journal Letters, vol. 628, no. 2, pp. L93-L96, 2005.

[81] I. Lee, M. Im, and Y. Urata, "First Korean observations of gamma-ray burst afterglows AT MT. Lemmon optical astronomy observatory (LOAO)," Journal of the Korean Astronomical Society, vol. 43, no. 3, pp. 95-104, 2010.

[82] Y. Urata, S. Nishiura, T. Miyata et al., "Multiband optical follow-up observations of GRB 020813 at the Kiso and Bisei observatories," The Astrophysical Journal, vol. 595, no. 1, pp. L21L24, 2003.

[83] R. S. De Souza, N. Yoshida, and K. Ioka, "Populations III.1 and III.2 gamma-ray bursts: constraints on the event rate for future radio and X-ray surveys," Astronomy and Astrophysics, vol. 533, article A32, 2011.

[84] H. Nagakura, Y. Suwa, and K. Ioka, "Population III gammaray bursts and breakout criteria for accretion-powered jets," The Astrophysical Journal, vol. 754, no. 2, article 85, 2012.

[85] D. Nakauchi, Y. Suwa, T. Sakamoto, K. Kashiyama, and T. Nakamura, "Long-duration X-ray flash and X-ray-rich gammaray bursts from low-mass population III stars," Astrophysical Journal, vol. 759, no. 2, article 9, 2012.

[86] S. Inoue, K. Omukai, and B. Ciardi, "The radio to infrared emission of very high redshift gamma-ray bursts: probing early star formation through molecular and atomic absorption lines," Monthly Notices of the Royal Astronomical Society, vol. 380, no. 4, pp. 1715-1728, 2007.

[87] D. Wanderman and T. Piran, "The luminosity function and the rate of Swift's gamma-ray bursts," Monthly Notices of the Royal Astronomical Society, vol. 406, no. 3, pp. 1944-1958, 2010.

[88] A. Cucchiara, A. J. Levan, D. B. Fox et al., "A photometric redshift of $z \sim 9.4$ for GRB 090429B," The Astrophysical Journal, vol. 736, no. 1, article 7, 2011.

[89] H. van Eerten, A. van der Horst, and A. MacFadyen, "Gammaray burst afterglow broadband fitting based directly on hydrodynamics simulations," The Astrophysical Journal, vol. 749, no. 1, article 44, 2012. 

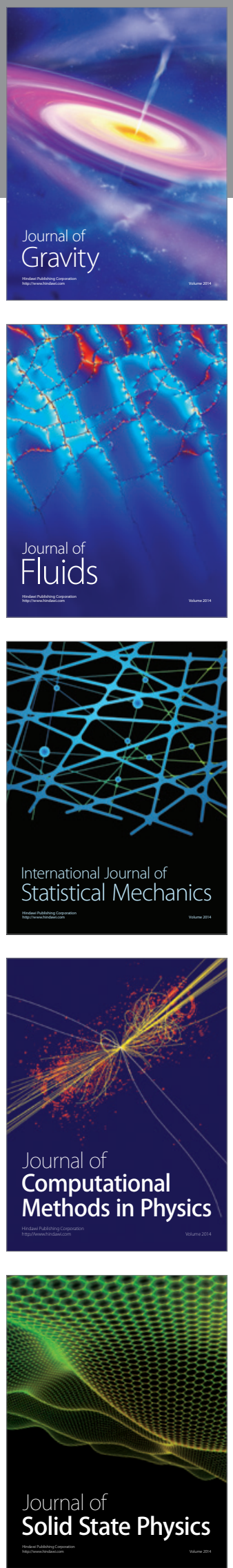

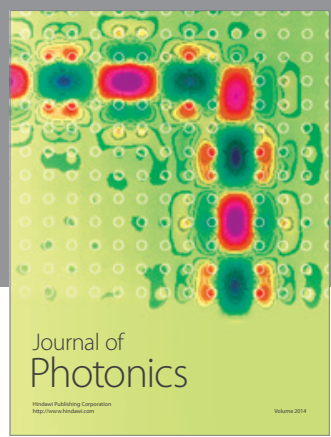

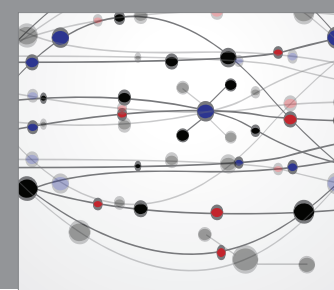

The Scientific World Journal

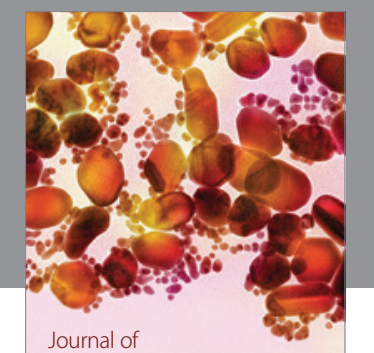

Soft Matter
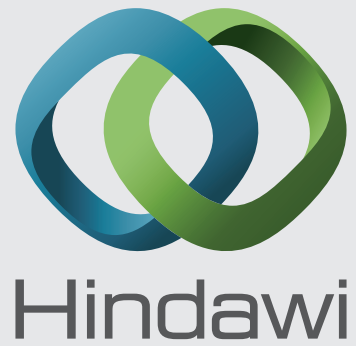

Submit your manuscripts at

http://www.hindawi.com
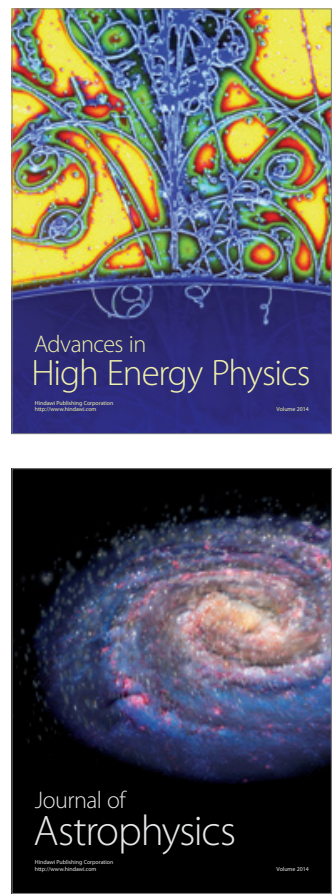
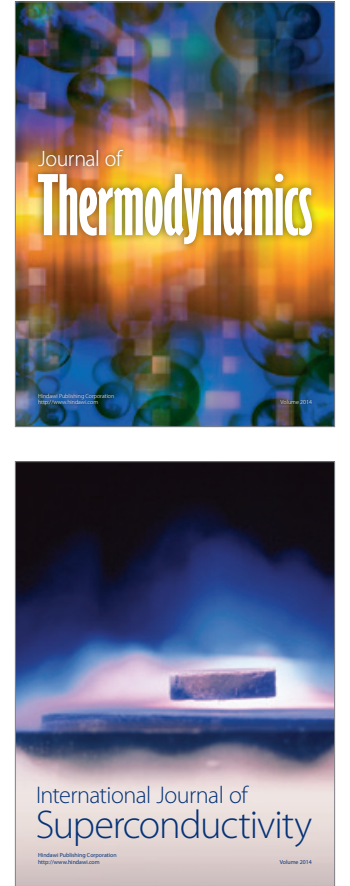
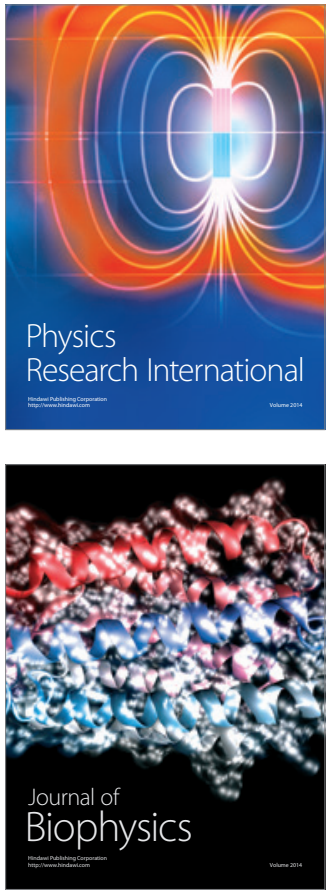
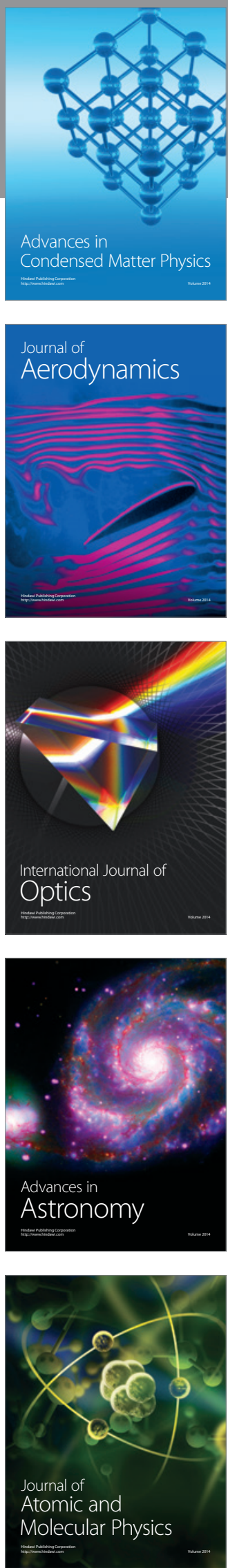